\title{
Physics and applications with laser-induced relativistic shock waves
}

\author{
S. Eliezer ${ }^{1,2}$, J. M. Martinez-Val ${ }^{1}$, Z. Henis ${ }^{2}$, N. Nissim², S. V. Pinhasi ${ }^{3}$, A. Ravid ${ }^{2}$, M. Werdiger ${ }^{2}$, and \\ E. Raicher ${ }^{2}$ \\ ${ }^{1}$ Institute of Nuclear Fusion, Polytechnic University of Madrid, Spain \\ ${ }^{2}$ Applied Physics Division, Soreq NRC Yavne, Israel \\ ${ }^{3} 42$ Beery, Rehovot, Israel \\ (Received 2 June 2016; revised 26 June 2016; accepted 8 July 2016)
}

\begin{abstract}
The laser-induced relativistic shock waves are described. The shock waves can be created directly by a high irradiance laser or indirectly by a laser acceleration of a foil that collides with a second static foil. A special case of interest is the creation of laser-induced fusion where the created alpha particles create a detonation wave. A novel application is suggested with the shock wave or the detonation wave to ignite a pre-compressed target. In particular, the deuteriumtritium fusion is considered. It is suggested that the collision of two laser accelerated foils might serve as a novel relativistic accelerator for bulk material collisions.
\end{abstract}

Keywords: fast ignition; laser piston model; relativistic shock waves; ultra-intense lasers

\section{Introduction}

From the time when Hugoniot ${ }^{[1]}$ completed the theory of shock waves in 1887 this subject is active ${ }^{[2,3]}$ and it is a basic field in many domains of science and applications. Since the passage time of the shock wave is short in comparison with the disassembly time of the shocked sample, one can do shock wave research for any pressure that can be supplied by a driver assuming that appropriate diagnostics are available. This fact enables, for example, thermodynamic measurements and equations of state (EOS) study at extremely high pressures and temperatures relevant for many domains of physics $^{[4,5]}$.

We are interested in a laser driver ${ }^{[6,7]}$ and in particular in very high irradiances ${ }^{[8,9]}, I_{L}>10^{21} \mathrm{~W} \mathrm{~cm}^{-2}$, in order to get relativistic shock waves. The theoretical foundation of relativistic shock waves is based on relativistic hydrodynamics ${ }^{[10]}$ and on the appropriate Hugoniot relations that were first analyzed by Taub ${ }^{[11]}$.

The interaction of a high power laser with a planar target creates a one-dimensional (1D) shock wave ${ }^{[7,12]}$. The theoretical basis for laser-induced shock waves analyzed and measured experimentally so far is based on plasma ablation. For laser intensities $10^{12} \mathrm{~W} \mathrm{~cm}^{-2}<I_{L}<10^{16} \mathrm{~W} \mathrm{~cm}^{-2}$

Correspondence to: Z. Henis. Applied Physics Division, Soreq NRC, Yavne 81800, Israel. Email: zoharhenis@ gmail.com and nanoseconds pulse duration hot plasma is created. This plasma exerts a high pressure on the surrounding material, leading to the formation of an intense shock wave moving into the interior of the target.

Using the ablation pressure in laser plasma interaction maximum pressures of the order of 1 Gbar $\left(=10^{9}\right.$ atmospheres), have been obtained during the collision of a target with an accelerating foil. In 1994 at the Livermore Laboratory in the USA this pressure was created by the impact of a gold foil accelerated with soft x-rays created from the Nova laser system ${ }^{[13]}$. Planar foil of polystyrene doped with $0.4 \%$ (atomic) bromine were accelerated by direct drive to $1000 \mathrm{~km} \mathrm{~s}^{-1}$ at the Institute of Laser Engineering at Osaka University in Japan ${ }^{[14]}$ creating upon impact a pressure of about 1 Gbar.

For the very high laser irradiances ultrahigh accelerations of the order of $10^{20} \mathrm{~cm} \mathrm{~s}^{-2}$ were predicted for high density plasma blocks ${ }^{[15-18]}$ created in sub-picosecond laser pulses with more than terawatt power. This effect was obtained by using two fluid simulations for laser plasma interaction where the nonlinear ponderomotive force was dominant ${ }^{[19,20]}$. Ion acceleration by ponderomotive force to relativistic velocities was also obtained using particles in cell simulations ${ }^{[21,22]}$. This acceleration is similar to the old proposal to accelerate space ships to relativistic velocities by laser pressure radiation ${ }^{[23]}$. 

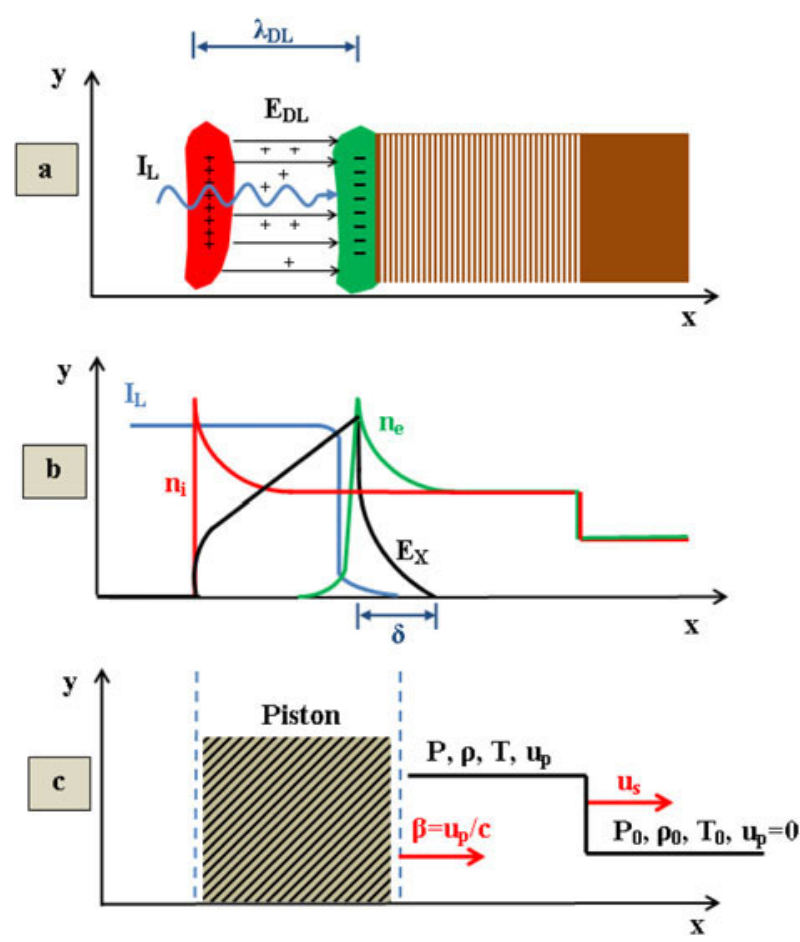

Figure 1. (a) Displays the capacitor model where the ponderomotive force dominates the interaction; (b) shows the DL of the negative and positive charges. (c) The shock wave description in the laboratory frame of reference.

The shock wave created in a 1D target by the ponderomotive force induced by very high laser irradiance, considered in this paper, is summarized schematically in Figure 1. In this domain of laser intensities the ponderomotive force accelerates the electrons forward, so that the charge separation field forms a double layer (DL), in which the ions are accelerated forward ${ }^{[24]}$. Figure 1(a) displays the capacitor model for laser irradiances $I_{L}$, where the ponderomotive force dominates the interaction and $\lambda_{\mathrm{DL}}$ is the distance between the positive and negative DL charges; Figure 1(b) shows the negative and positive layers where $n_{e}$ and $n_{i}$ are the electron and ion densities accordingly, $E_{x}$ is the electric field, and $\delta$ is the solid density skin depth of the foil. The shock wave description in the laboratory frame of reference is given in Figure 1(c). This DL acts as a piston driving a shock wave ${ }^{[25,26]}$, moving in the unperturbed plasma. This plasma has in general different ion and electron temperatures.

In Section 2, we summarize the relativistic formalism for shock waves in solid targets. Section 3 is designated to calculate the laser acceleration of a micro-foil and the shock wave created when this foil collides with a static target. The fast ignition of deuterium-tritium (DT) fuel is given in Section 4. In Section 5, the possibility to ignite a DT fuel by a nuclear detonation wave is considered. We conclude with a summary and perspective in Section 6.

\section{Laser-induced relativistic shock wave}

A relativistic or non-relativistic ${ }^{[3]}$ shock wave is described in the 1D geometry by five variables: particle density $n$ (or the density $\rho=M n$ where $M$ is the particle mass), the pressure $P$, the energy density $e$, the shock wave velocity $u_{s}$ and the particle flow velocity $u_{p}$, assuming that we know the initial condition of the target $\left(\rho_{0}, P_{0}, e_{0}\right.$ and the particle flow velocity $u_{0}$ ) before the shock arrival. The four equations relating the shock wave variables are the three Hugoniot relations describing the conservation laws of energy, momentum and particles and the EOS connecting the thermodynamic variables of the state under consideration ${ }^{[3-5]}$. The fifth equation necessary to solve the problem is obtained in a model ${ }^{[26]}$ where the pressure is induced by the laser ponderomotive force and its strength is a function of the laser pulse parameters ${ }^{[27]}$.

Note that in the general 3-dimensional (3D) shock wave case one has seven variables since velocities are a $3 \mathrm{D}$ vector. In this case there are six Hugoniot relations describing the conservation laws of energy, momentum and particles and the EOS. The last equation is model dependent, or if possible, is preferable that one parameter is measured experimentally.

The relativistic hydrodynamic starting point is the energymomentum 4-tensor $T_{\mu \nu}$ given by

$$
T_{\mu \nu}=(e+P) U_{\mu} U_{\nu}+P g_{\mu \nu},
$$

where $U_{\mu}(\mu=0,1,2,3)$ is the dimensionless 4-velocity in which the subscripts 0 is the time component and $(1,2,3)$ are the space $(x, y, z)$ components accordingly, and $g_{\mu \nu}$ is the metric tensor,

$$
\begin{gathered}
c U_{\mu}=\left(\gamma c, \gamma v_{1}, \gamma v_{2}, \gamma v_{3}\right), \\
g_{\mu \nu}: g_{00}=-1, \quad g_{11}=g_{22}=g_{33}=1, \\
g_{\mu \nu}=0 \quad \text { if } \mu \neq v, \\
\gamma=\frac{1}{\sqrt{1-\beta^{2}}} ; \quad \beta=\frac{v}{c} ; \quad v=\sqrt{v_{1}^{2}+v_{2}^{2}+v_{3}^{2}},
\end{gathered}
$$

where $c$ is the speed of light. The energy-momentum conservation, the particle number conservation and the EOS are given accordingly (Einstein summation is assumed from 0 to 3 for identical indexes)

$$
\begin{aligned}
\frac{\partial T_{\mu}^{\nu}}{\partial x^{\nu}} \equiv \partial_{\nu} T_{\mu}^{\nu} & =0 \quad \text { for } \mu=0,1,2,3, \\
\frac{\partial\left(n U^{\mu}\right)}{\partial x^{\mu}} & \equiv \partial_{\mu}\left(n U^{\mu}\right)=0, \\
P & =P(e, n) .
\end{aligned}
$$

The EOS taken here in order to calculate the shock wave parameters is the ideal gas EOS 


$$
e=\rho c^{2}+\frac{P}{\Gamma-1}
$$

where $\Gamma$ is the specific heat ratio of constant pressure to constant volume. Writing explicitly Equations (3) with the ideal gas EOS Equation (4) in the laboratory frame of reference yield

$$
\begin{aligned}
& \text { (i) } \frac{u_{p 1}}{c}=\sqrt{\frac{\left(P_{1}-P_{0}\right)\left(e_{1}-e_{0}\right)}{\left(e_{0}+P_{1}\right)\left(e_{1}+P_{0}\right)}}, \\
& \text { (ii) } \frac{u_{s}}{c}=\sqrt{\frac{\left(P_{1}-P_{0}\right)\left(e_{1}+P_{0}\right)}{\left(e_{1}-e_{0}\right)\left(e_{0}+P_{1}\right)}}, \\
& \text { (iii) } \frac{\left(e_{1}+P_{1}\right)^{2}}{\rho_{1}^{2}}-\frac{\left(e_{0}+P_{0}\right)^{2}}{\rho_{0}^{2}} \\
& =\left(P_{1}-P_{0}\right)\left[\frac{\left(e_{0}+P_{0}\right)}{\rho_{0}^{2}}+\frac{\left(e_{1}+P_{1}\right)}{\rho_{1}^{2}}\right], \\
& \text { (iv) } e_{j}=\rho_{j} c^{2}+\frac{P_{j}}{\Gamma-1} ; \quad j=0,1 .
\end{aligned}
$$

The subscripts 0 and 1 define the flow and thermodynamic parameters before and after shock wave arrival accordingly and $u_{p 0}=u_{o}=0$ has been assumed in this case.

The relativistic shock wave of Equations (5) with $e=$ $\rho c^{2}+\rho E$, where $P$ and $\rho E$ are much smaller than $\rho c^{2}$, the velocities $v$ satisfy $v / c \ll 1$, yield the following nonrelativistic well-known Hugoniot equations,

$$
\text { (i) } u_{p 1}=\left(1 / \rho_{0}-1 / \rho_{1}\right)^{1 / 2}\left(P_{1}-P_{0}\right)^{1 / 2} \text {, }
$$

(ii) $u_{s}=\left(1 / \rho_{0}\right)\left(1 / \rho_{0}-1 / \rho_{1}\right)^{-1 / 2}\left(P_{1}-P_{0}\right)^{1 / 2}$,

(iii) $E_{1}-E_{0}=(1 / 2)\left(1 / \rho_{0}-1 / \rho_{1}\right)\left(P_{1}+P_{0}\right)$,

$$
\text { (iv) } E_{j}=\left(\frac{1}{\Gamma-1}\right)\left(\frac{P_{j}}{\rho_{j}}\right) \quad \text { for } j=0,1 \text {. }
$$

For the relativistic case we have to solve Equations (5) together with the piston model equation ${ }^{[21,27]}$

$$
P_{1}=\frac{2 I_{L}}{c}\left(\frac{1-\beta}{1+\beta}\right) ; \quad \beta \equiv \frac{u_{p 1}}{c} .
$$

Equations (5) and (7) are five equations with five unknowns: $u_{s}, u_{p 1}, P_{1}, \rho_{1}, e_{1}$ assuming that we know $I_{L}, \rho_{0}, P_{0}, \Gamma$ and $u_{o}=0$. The calculations are conveniently done in the dimensionless units defined by

$$
\begin{aligned}
\Pi_{L} & \equiv \frac{I_{L}}{\rho_{0} c^{3}} ; \quad \kappa \equiv \frac{\rho_{1}}{\rho_{0}} ; \quad \kappa_{0} \equiv \frac{\Gamma+1}{\Gamma-1} ; \quad \Pi=\frac{P_{1}}{\rho_{0} c^{2}} \\
\Pi_{0} & =\frac{P_{0}}{\rho_{0} c^{2}} .
\end{aligned}
$$

Substituting the ideal gas EOS into the third of Equations (5) we get the relativistic Hugoniot equation

$$
\begin{gathered}
\left\{\begin{array}{l}
\Pi^{2}+B \Pi+C=0 \\
\kappa \geqslant 1,
\end{array}\right. \\
\Pi=\left(\frac{1}{2}\right)\left(-B \pm \sqrt{B^{2}-4 C}\right), \\
B=\frac{(\Gamma-1)^{2}}{\Gamma}\left(\kappa_{0} \kappa-\kappa^{2}\right)+\Pi_{0}(\Gamma-1)\left(1-\kappa^{2}\right), \\
C=\frac{(\Gamma-1)^{2}}{\Gamma}\left(\kappa-\kappa_{0} \kappa^{2}\right) \Pi_{0}-\kappa^{2} \Pi_{0}^{2} .
\end{gathered}
$$

It is important to emphasize that if we take $P_{0}=0$ then we get only the $\kappa>\kappa_{0}$ solutions.

The relativistic Hugoniot equation for an ideal gas EOS is given by Equations (9) while the non-relativistic Hugoniot equation for an ideal gas EOS is (from Equations (6))

$$
\Pi=\left(\frac{\kappa \kappa_{0}-1}{\kappa_{0}-\kappa}\right) \Pi_{0} .
$$

Figure 2 describes the transition between the relativistic and non-relativistic Hugoniot, namely the transition between Equations (10) and (9). In this transition domain, between relativistic and non-relativistic shock waves, we have

$$
\begin{aligned}
10^{-9} \leqslant \Pi \leqslant 10^{-2} \Leftrightarrow \kappa & =\frac{\rho}{\rho_{0}} \\
& =\frac{\Gamma+1}{\Gamma-1}(=4.00 \text { for } \Gamma=5 / 3) .
\end{aligned}
$$

In the domain defined by Equation (11) we can use the first two equations of Equations (6) for $u_{p 1} / c<0.03$ in order to get

$$
\begin{gathered}
\frac{u_{p 1}}{c}=\sqrt{\frac{2 \Pi}{\Gamma+1}} ; \\
\frac{u_{s}}{c}=\sqrt{\frac{(\Gamma+1) \Pi}{2}} .
\end{gathered}
$$

Using now our piston model Equations (7) together with Equations (12) we obtain in the intermediate domain between relativistic and non-relativistic shock waves

$$
\begin{gathered}
\beta=\frac{u_{p 1}}{c}=\frac{-\Pi_{L}+\sqrt{\left(\frac{\Gamma+1}{4}\right) \Pi_{L}-\Pi_{L}^{2}}}{\left(\frac{\Gamma+1}{4}\right)-2 \Pi_{L}}, \\
\Pi=2 \Pi_{L}\left[\frac{\left(\frac{\Gamma+1}{4}\right)-\Pi_{L}-\sqrt{\left(\frac{\Gamma+1}{4}\right) \Pi_{L}-\Pi_{L}^{2}}}{\left(\frac{\Gamma+1}{4}\right)-3 \Pi_{L}+\sqrt{\left(\frac{\Gamma+1}{4}\right) \Pi_{L}-\Pi_{L}^{2}}}\right] .
\end{gathered}
$$

Since $\Pi_{L}<10^{-2}$ we have to a good approximation in the domain between relativistic and non-relativistic shock wave 


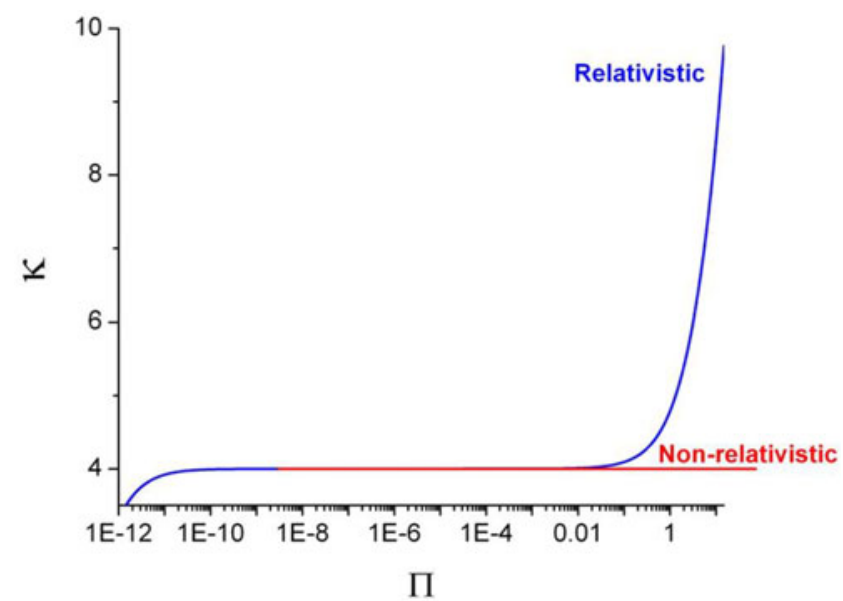

Figure 2. The shock wave compression $\kappa=\rho / \rho_{0}$ as a function of the dimensionless shock wave pressure $\Pi=P / \rho_{0} c^{2}$ for $\Gamma=5 / 3$.

the following approximations

$$
\begin{gathered}
\Pi \approx 2 \Pi_{L} \Rightarrow P=\frac{2 I_{L}}{c} \\
\frac{u_{p 1}}{c} \approx 2 \sqrt{\frac{\Pi_{L}}{\Gamma+1}}=2 \sqrt{\frac{I_{L}}{(\Gamma+1) \rho_{0} c^{3}}} \\
\frac{u_{s}}{c} \approx \sqrt{(\Gamma+1) \Pi_{L}}=\sqrt{\frac{I_{L}(\Gamma+1)}{\rho_{0} c^{3}}}
\end{gathered}
$$

Under this approximation, the shock wave length $l_{s}$ by the end of the laser pulse $\tau_{L}$ is

$$
l_{s}=\left(u_{s}-u_{p 1}\right) \tau_{L}=(\Gamma-1) c \tau_{L} \sqrt{\frac{\Pi_{L}}{(\Gamma+1)}} .
$$

The laser cross-section $S_{L}=\pi R_{L}^{2}$ is chosen $R_{L}=1.5\left(u_{s}-\right.$ $\left.u_{p 1}\right) \tau_{L}$ in order that the $1 \mathrm{D}$ laser-induced shock wave is conceivable. Therefore, for a constant laser irradiation $I_{L}$ we need a laser energy $W_{L}$ given by

$$
W_{L}=I_{L} S_{L} \tau_{L}=2.25 \pi\left[\frac{(\Gamma-1)^{2}}{\Gamma+1}\right]\left(\frac{I_{L}^{2} \tau_{L}^{3}}{\rho_{0} c}\right)
$$

The numerical solutions of the exact relativistic Equations (5) and (7) are given in Figures 3 and 4. Figure 3 gives the Hugoniot dimensionless shock wave pressure $\Pi=P /\left(\rho_{0} c^{2}\right)$ versus the dimensionless laser irradiance $\Pi_{L}=I_{L} /\left(\rho_{0} c^{3}\right)$ in the domain $10^{-4}<\Pi_{L}<1$. For a better understanding of this graph and for the practical proposal in the next section, the inserted table shows numerical values in the area $10^{-4}<\Pi_{L}<10^{-2}$. Figure 4 describes the dimensionless shock wave velocity $u_{s} / c$ and the particle velocity $u_{p} / c\left(u_{p 1} \equiv u_{p}\right)$ in the laboratory frame of reference versus the dimensionless laser irradiance

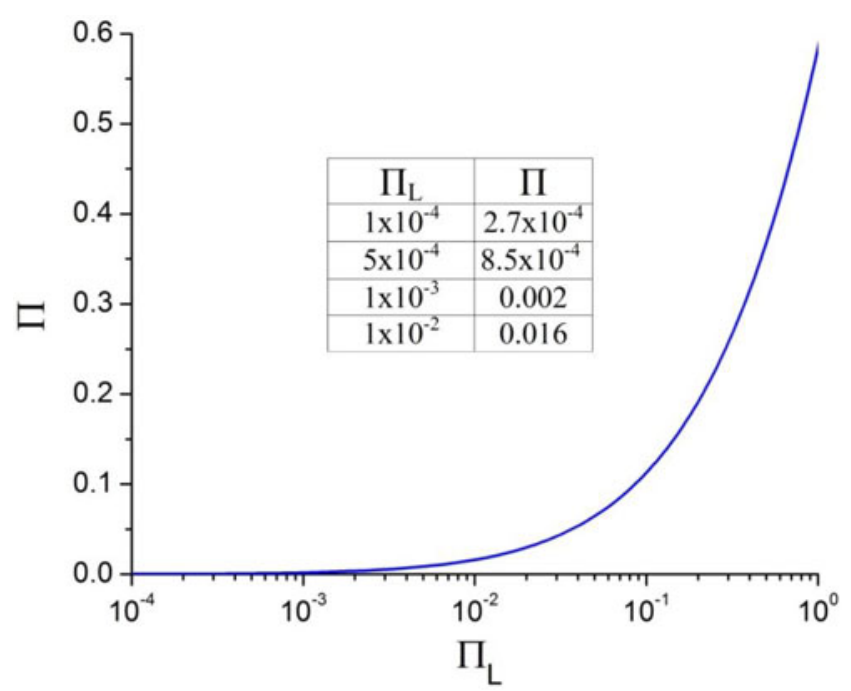

Figure 3. The dimensionless shock wave pressure $\Pi=P /\left(\rho_{0} c^{2}\right)$ versus the dimensionless laser irradiance $\Pi_{L}=I_{L} /\left(\rho_{0} c^{3}\right)$ in the domain $10^{-4}<$ $\Pi_{L}<1$. The inserted table shows numerical values in the area $10^{-4}<$ $\Pi_{L}<10^{-2}$.

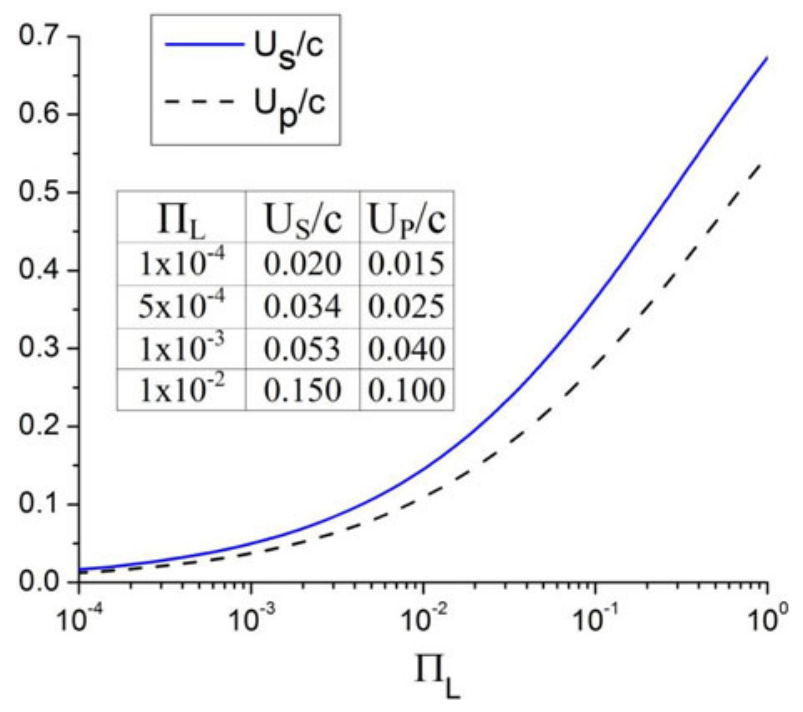

Figure 4. The dimensionless shock wave velocity $u_{S} / c$ and the particle velocity $u_{p} / c$ in the laboratory frame of reference versus the dimensionless laser irradiance $\Pi_{L}=I_{L} /\left(\rho_{0} c^{3}\right)$ in the domain $10^{-4}<\Pi_{L}<1$. The inserted table shows numerical values in the area $10^{-4}<\Pi_{L}<10^{-2}$.

$\Pi_{L}=I_{L} /\left(\rho_{0} c^{3}\right)$ in the domain $10^{-4}<\Pi_{L}<1$, while the inserted table shows numerical values in the area $10^{-4}<$ $\Pi_{L}<10^{-2}$. As a numerical example we take a target with initial density $\rho_{0}=1 \mathrm{~g} \mathrm{~cm}^{-3}$ irradiated by a laser with intensity $I_{L}=5 \times 10^{23} \mathrm{~W} \mathrm{~cm}^{-2}$, namely $\Pi_{L}=0.185$. In this case our relativistic equations yield a compression $\kappa=\rho / \rho_{0}=4.09$, a pressure $P=3.3 \times 10^{14}$ bars, a shock wave velocity $u_{s}=0.35 c$ and a particle velocity $u_{p}=0.27 c$ where $c$ is the speed of light. 
The relativistic speed of sound $c_{S}$ ( $S$ is entropy) for an ideal gas EOS is

$$
\frac{c_{s}}{c}=\sqrt{\left(\frac{\partial P}{\partial e}\right)_{S}}=\left(\frac{\Gamma P}{e+P}\right)^{1 / 2}=\left[\frac{\Gamma(\Gamma-1) \Pi}{\Gamma \Pi+(\Gamma-1) \kappa}\right]^{1 / 2} .
$$

In the shocked medium the characteristic velocity of a disturbance from the piston to the shock wave front equals the rarefaction wave $c_{r w}$ and is given by

$$
c_{r w}=\frac{c_{S}+u_{p}}{1+\left(\frac{c_{S} u_{p}}{c^{2}}\right)} .
$$

Figures 5(a) and 5(b) describe accordingly the speed of sound in units of speed of light, $c_{S} / c$, and the ratio of shock velocity to the rarefaction velocity, $u_{s} / c_{r w}$, as a function of the dimensionless laser irradiance $\Pi_{L}=I_{L} /\left(\rho_{0} c^{3}\right)$ in the domain $10^{-4}<\Pi_{L}<1$. The inserted tables show numerical values for $10^{-4}<\Pi_{L}<10^{-2}$.

From Figures 4 and 5 (and the associated relativistic equations describing these values) one can see that the necessary conditions for a 1D shock wave stability are satisfied. First the speed of sound increases with increasing pressure (see Equation (17)). Secondly, a disturbance behind the shock wave front cannot be slower than the shock velocity, because in this case it will not be able to catch the wave front and the shock would decay (i.e., unstable). Thirdly, a small compressive disturbance ahead of the shock wave must move slower than the shock front in order not to create another shock wave.

\section{Laser relativistic acceleration of a micro-foil and the shock wave created by impact on a secondary foil}

In this section, we first calculate the high power laser acceleration of a micro-foil ${ }^{[23,28]}$ and secondly the shock waves created upon impact between the accelerated foil and a static target.

Physical quantities in the instantaneous rest frame of reference of the micro-foil are denoted by the subscript $F$ while their laboratory frame of reference values are written without any subscript. For a laser irradiance $I_{F}\left[\mathrm{erg} /\left(\mathrm{cm}^{2} \mathrm{~s}\right)\right]=$ $c E_{F}^{2} /(4 \pi)$, where $E_{F}$ is the laser electromagnetic field in the foil frame of reference, the radiation pressure is equal to the ponderomotive pressure given by

$$
P=P_{F}=\frac{I_{F}}{c}\left(1+R_{F}-T_{F}\right)=\frac{2 I_{F} R_{F}}{c},
$$

where $R_{F}$ is the reflected laser and $T_{F}$ is the transmitted laser through the foil which has to be taken into account for thin foils and very high laser irradiances. The right hand side of Equation (19) is obtained assuming the energy conservation
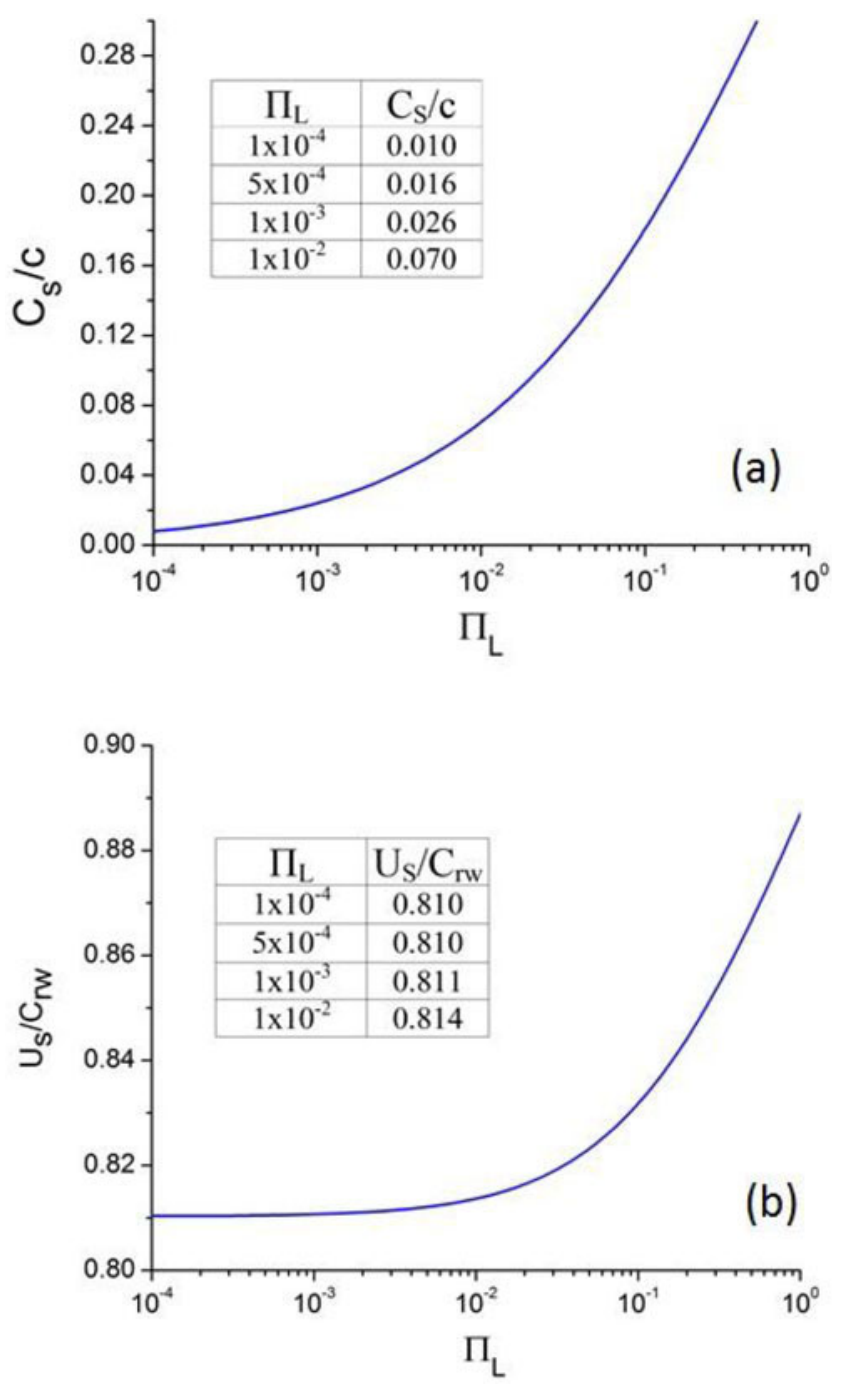

Figure 5. (a) The speed of sound in units of speed of light, $c_{S} / c$ and (b) the ratio of shock velocity to the rarefaction velocity, $u_{S} / c_{r w}$ as a function of the dimensionless laser irradiance $\Pi_{L}=I_{L} /\left(\rho_{0} c^{3}\right)$.

equation: $R_{F}+T_{F}=1$. Note that in this equation we have used the fact that the radiation pressure $P=P_{F}$ if the foil is moving in the laboratory frame of reference in the $x$ direction since for the force one has $F_{x}=\left(F_{F}\right)_{x}$. The laboratory laser irradiance $I$ is related to the laser irradiance in the micro-foil rest frame $I_{F}$ through the Doppler effect (DE)

$$
I=I_{F}\left(\frac{\omega}{\omega_{F}}\right)^{2}=I_{F}\left(\frac{1+\beta_{f}}{1-\beta_{f}}\right) ; \quad \beta_{f}=\frac{u_{f}}{c},
$$

where $u_{f}$ is the micro-foil velocity in the laboratory. Using Equations (19) and (20) for $R_{F}=1$ we get the ponderomotive pressure as a function of laboratory frame quantities

$$
P=\frac{2 I}{c}\left(\frac{1-\beta_{f}}{1+\beta_{f}}\right) .
$$



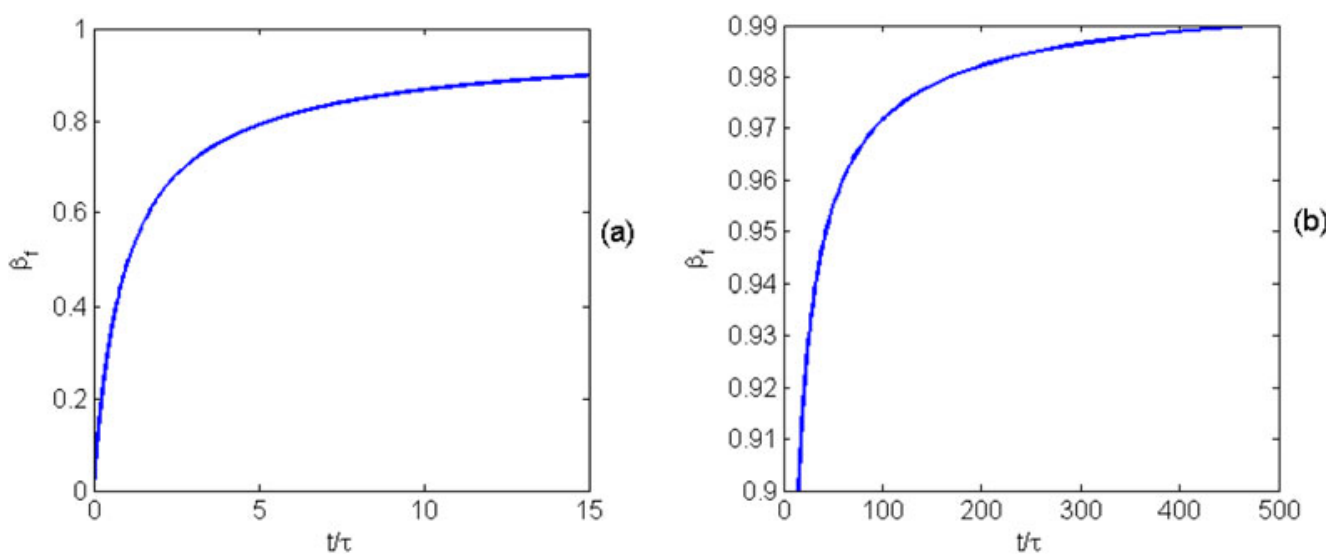

Figure 6. Micro-foil velocity as a function of laser pulse duration $t$ in units of of $\tau=\rho_{0} c^{2} l /(2 I)$, where $\rho_{0}$ is the initial density, $l$ is the foil thickness and $I$ is the laser intensity [erg/( $\left.\left.\mathrm{s} \mathrm{cm}^{2}\right)\right]$. (a) Laser pulse duration up to $15 \tau$, (b) laser pulse duration up to $500 \tau$.

This equation is identical to the piston model velocity of the particle flow velocity as given in Equations (7). The Newton law of motion for the foil in the laboratory frame of reference is

$$
\begin{gathered}
\frac{d p_{f}}{d t}=P S \Rightarrow \\
\frac{d}{d t}\left[\left(\rho_{0} l c\right) \frac{\beta_{f}}{\sqrt{1-\beta_{f}^{2}}}\right]=\frac{2 I}{c}\left(\frac{1-\beta_{f}}{1+\beta_{f}}\right) \Rightarrow \\
\frac{1}{\left(1+\beta_{f}\right)^{1 / 2}\left(1-\beta_{f}\right)^{5 / 2}} \frac{d \beta_{f}}{d t}=\frac{2 I}{\rho_{0} c^{2} l},
\end{gathered}
$$

in which we have used the momentum of the micro-foil $p_{f}=$ $M_{0 f} \gamma_{f} \beta_{f} c$ where $\gamma_{f}=\left(1-\beta_{f}^{2}\right)^{-1 / 2}$ and the foil rest mass is $M_{0 f}=\rho_{0} S l$ where $\rho_{0}$ is the initial density, $S$ the crosssection area and $l$ the thickness of the micro-foil. The force accelerating the micro-foil is $F=P S$. For constant $I$ this equation is easily integrated

$$
\begin{aligned}
\int_{0}^{\beta_{f}} \frac{d x}{(1-x)^{5 / 2}(1+x)^{1 / 2}} & =\frac{\left(2-\beta_{f}\right) \sqrt{1-\beta_{f}^{2}}}{3\left(1-\beta_{f}\right)^{2}}-\frac{2}{3} \\
& =\frac{2 I t}{\rho_{0} c^{2} l} \equiv \frac{t}{\tau}
\end{aligned}
$$

From this solution one can see that $\beta_{f}(t / \tau) \rightarrow 1$ for $t / \tau \rightarrow$ $\infty$, namely the relativistic velocities are obtained if $t / \tau \gg 1$ which implies a laser pulse duration much larger than $\tau=$ $\rho_{0} c^{2} l /(2 I)$ or equivalently $I t \gg \rho_{0} c^{2} l / 2$. For an initial density $\rho_{0}=1 \mathrm{~g} \mathrm{~cm}^{-3}, l=0.1 \mu \mathrm{m}$ one gets the scaling time $\tau=45$ and 0.45 fs for $I=10^{22}$ and $10^{24} \mathrm{~W} \mathrm{~cm}^{-2}$ accordingly. The micro-foil velocity as a function of the laser pulse duration defined by $t$ in this case is described in Figure 6. The time scale in Figure 6 is $\tau=\rho_{0} c^{2} l /(2 I)$, where $\rho_{0}$ is the initial density, $l$ is the foil thickness and $I$ is the laser intensity $\left[\mathrm{erg} /\left(\mathrm{s} \mathrm{cm}^{2}\right)\right]$. Figure 6(a) describes the accelerated foil velocity $\beta_{f}$ for laser pulse durations up to
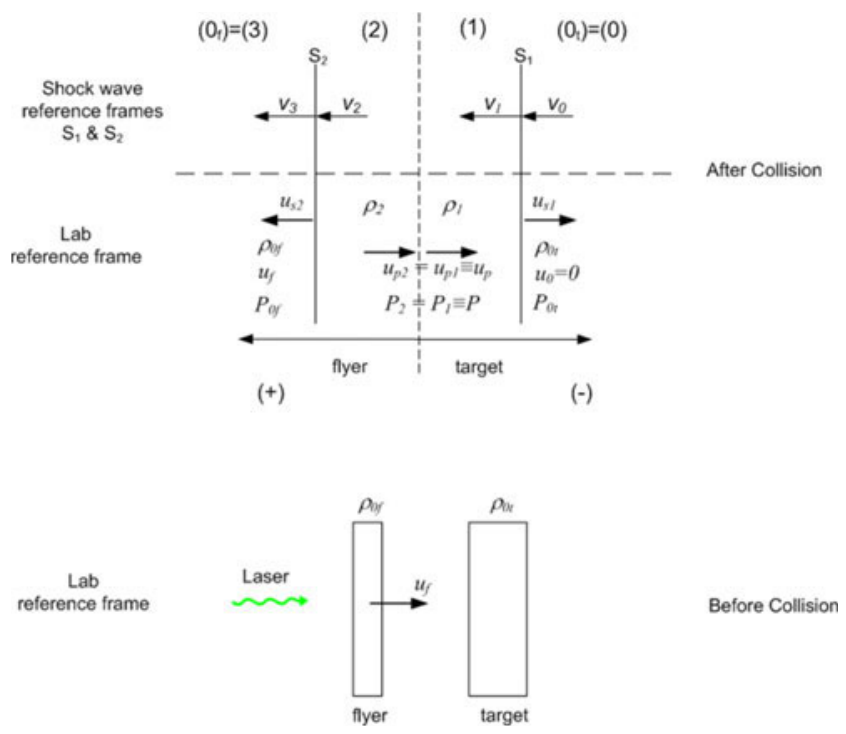

Figure 7. Flow $\left(u_{p 0}, u_{p 1}=u_{p 2}\right)$ and shock waves $\left(u_{s 1}, u_{s 2}\right)$ velocities after impact of flyer and target in the laboratory frame of reference. The flow velocities $\left(v_{0}, v_{1}, v_{2}=v_{1}, v_{3}=v_{0}\right)$ are also defined in the shock wave reference frames S1 and S2. The lower figure shows a schematic picture before collision.

$15 \tau$ while Figure 6(b) gives $\beta_{f}$ as a function of the laser pulse durations up to $500 \tau$.

The shock waves upon impact of this accelerated foil with a static target are now calculated. The shock waves variables are defined in Figure 7. The flow particle velocities $\left(u_{p 0}=\right.$ $\left.u_{0}=0, u_{p 1}=u_{p 2} \equiv u_{p}\right)$ and the shock wave velocities $\left(u_{s 1}\right.$, $u_{s 2}$ ) after impact of the flyer and the target in the laboratory frame of reference are pointed out in this figure. The flow velocities $\left(v_{0}, v_{1}, v_{2}=v_{1}, v_{3}=v_{0}\right)$ are also defined in the shock wave reference frames S1 and S2. The lower figure shows a schematic picture before collision.

The flyer has a known initial (before impact) velocity $u_{f}$ in the laboratory frame of reference. This velocity can be calculated from Equation (23) and it is possible to measure 
it experimentally by using the relativistic $\mathrm{DE}$

$$
\text { longitudinal DE: } \frac{\lambda_{o}}{\lambda_{s}}=\frac{v_{s}}{v_{o}}=\sqrt{\frac{1+\beta_{f}}{1-\beta_{f}}} ; \beta_{f}=\frac{u_{f}}{c} \text {, }
$$

transversal DE: $\frac{v_{s}}{v_{o}}=\gamma_{f}\left(1+\beta_{f} \cos \theta_{o}\right) ; \quad \gamma_{f}=\frac{1}{\sqrt{1-\beta_{f}^{2}}}$,

where $s$ and $o$ refer to observer and source frames of reference accordingly, $\theta_{o}$ is the angle between the flyer motion and the observer, $\lambda$ and $v$ are the appropriate wavelength and frequency of the electromagnetic wave. It is interesting and might be experimentally useful to see the relativistic effect $v_{s} / \nu_{o}\left(\theta_{o}=\pi / 2\right)=\gamma_{f}$.

First we solve the relativistic symmetrical collision, namely the target and the flyer are the same material. If the 0 domain of the target is at rest in the laboratory frame of reference, then in this frame of reference the shock velocity $u_{s}=-v_{0}$. (Note that $v_{0}>0$ and $u_{s}<0$ means that the positive $x$ coordinate is defined toward the back of the shock wave.) Using the definitions, $\beta_{0}=v_{0} / c, \beta_{1}=v_{1} / c$ and $\beta_{f}=u_{f} / c$ where $u_{f}$ is the flyer velocity in the laboratory frame of reference while $v_{0}$ and $v_{1}$ are the flow velocities in the shock wave frames of reference $S 1$ and $S 2$, we get the shock waves velocities in the laboratory frame of reference by using Equations (5) and relativistic addition of velocities

$$
\begin{gathered}
\frac{u_{s 1}}{c}=-\beta_{0}=-\sqrt{\frac{\left(P_{1}-P_{0}\right)\left(e_{1}+P_{0}\right)}{\left(e_{1}-e_{0}\right)\left(e_{0}+P_{1}\right)}}, \\
\frac{u_{s 2}}{c}=\frac{\beta_{1}+\beta_{f}}{1+\beta_{1} \beta_{f}}=\frac{1+\beta_{f} \sqrt{\frac{\left(e_{1}-e_{0}\right)\left(e_{1}+P_{0}\right)}{\left(P_{1}-P_{0}\right)\left(e_{0}+P_{1}\right)}}}{\beta_{f}+\sqrt{\frac{\left(e_{1}-e_{0}\right)\left(e_{1}+P_{0}\right)}{\left(P_{1}-P_{0}\right)\left(e_{0}+P_{1}\right)}}} .
\end{gathered}
$$

In the non-relativistic case, the Galilean transformations yield for the particle velocity in the laboratory frame $u_{p 1}=\left(v_{1}-v_{0}\right)$ at the S1 surface singularity and $u_{p 1}=$ $-\left(v_{1}-v_{0}\right)+u_{f}$ at the S2 surface singularity. From these two equations we get the well-known result: $u_{f}=2 u_{p 1}$. We now use the same procedure by using the relativistic Lorentz transformation.

From Equations (5) we get at the S1 surface singularity the particle flow velocity $u_{p 1}$ in the shocked area in the laboratory frame of reference

$$
u_{p 1}=v_{01}=-c \sqrt{\frac{\left(P_{1}-P_{0}\right)\left(e_{1}-e_{0}\right)}{\left(e_{0}+P_{1}\right)\left(e_{1}+P_{0}\right)}} .
$$

On the other hand, at the S2 surface singularity the particle flow velocity $u_{p 1}=u_{p 2}$ in the shocked area in the laboratory frame of reference is

$$
u_{p 1}=\frac{v_{01}-u_{f}}{1-v_{01} u_{f} / c^{2}}
$$

Equations (26) and (27) we get

$$
\sqrt{\frac{\left(P_{1}-P_{0}\right)\left(e_{1}-e_{0}\right)}{\left(e_{0}+P_{1}\right)\left(e_{1}+P_{0}\right)}}=\frac{1-\sqrt{1-\beta_{f}^{2}}}{\beta_{f}} .
$$

This relation yields the known result $u_{f}=2 u_{p 1}$ in the nonrelativistic limit.

We calculate $e_{1}, P_{1}$ and $\rho_{1}$ as a function of $u_{f}$. The initial conditions for our suggested impact are: $P_{0}, e_{0}=\rho_{0} c^{2}+$ $P_{0} /(\Gamma-1)$ for an initial density $\rho_{0}$. Since in the impact under consideration the initial pressure is extremely small we take $P_{0}=0$. This formalism implies the following solutions

$$
\begin{gathered}
\frac{P_{1}}{\rho_{0} c^{2}}=\frac{1-\beta_{f}^{2} / 3-\sqrt{1-\beta_{f}^{2}}}{-1+\beta_{f}^{2}+\sqrt{1-\beta_{f}^{2}}} \\
\frac{P_{1}}{\rho_{0} c^{2}}=\left(\frac{(\Gamma-1)^{2}}{\Gamma}\right)\left(\frac{\rho_{1}}{\rho_{0}}\right)^{2}-\left(\frac{\Gamma^{2}-1}{\Gamma}\right)\left(\frac{\rho_{1}}{\rho_{0}}\right) .
\end{gathered}
$$

Now the relativistic asymmetrical collision is discussed, namely the target and the flyer do not have the same initial densities. Here it is also assumed that we can neglect the initial pressure, namely we take $P_{0}=0$. This constrain yields compressions greater than $(\Gamma+1) /(\Gamma-1)$ as stated before.

The input data for our problem is: target initial flow velocity $u_{0}=0$, initial densities $\rho_{0 t}$ and $\rho_{0 f}$ and initial pressures $P_{0 t} P_{0 f}$ of target and flyer accordingly and target and flyer EOS parameters are appropriately $\Gamma_{t}, \Gamma_{f}$. All variables are described as a function of the foil velocity $\beta_{f}$ that is measured experimentally. There are eight unknowns- $\rho_{1}$, $\rho_{2}, u_{s 1}, u_{s 2}, u_{p 1}, u_{p 2}, P_{1}$ and $P_{2}$ with eight equations: The two Hugoniot relations for target and flyer (indices 1 and 2 accordingly), four Hugoniot equations describing the mass and momentum conservations for target and flyer that yield the particle velocities $u_{p 1}$ and $u_{p 2}$ and the shock velocities $u_{s 1}$ and $u_{s 2}$. These six equations yield

$$
\begin{gathered}
u_{p 1}=-c \sqrt{I_{1}} ; \quad u_{p 2}=-c\left(\beta_{f}-\sqrt{I_{2}}\right)\left(1-\beta_{f} \sqrt{I_{2}}\right)^{-1}, \\
u_{s 1}=-c \sqrt{J_{1}} ; \quad u_{s 2}=-c\left(\beta_{f}-\sqrt{J_{2}}\right)\left(1-\beta_{f} \sqrt{J_{2}}\right)^{-1}, \\
I_{i}=\frac{\Pi_{i}\left[\Pi_{i}+(2 / 3)\left(\kappa_{i}-1\right)\right]}{\left(\Pi_{i}+1\right)\left[\Pi_{i}+(2 / 3) \kappa_{i}\right]} \quad \text { for } i=1,2, \\
J_{i}=\frac{\Pi_{i}\left[(2 / 3) \kappa_{i}+\Pi_{i}\right]}{\left[(2 / 3)\left(\kappa_{i}-1\right)+\Pi_{i}\right]\left[1+\Pi_{i}\right]} \quad \text { for } i=1,2 .
\end{gathered}
$$

Finally, the following two continuum equations at the impact between flyer and target are:

$$
\begin{gathered}
P_{1}=P_{2} \Rightarrow \kappa_{2}^{2}-4 \kappa_{2}=K\left[\kappa_{1}^{2}-4 \kappa_{1}\right] \\
u_{p 1}=u_{p 2} \Rightarrow-\sqrt{I_{1}}+\beta_{f} \sqrt{I_{1} I_{2}}=\sqrt{I_{2}}-\beta_{f}, \\
K \equiv \rho_{0 t} / \rho_{0 f} .
\end{gathered}
$$



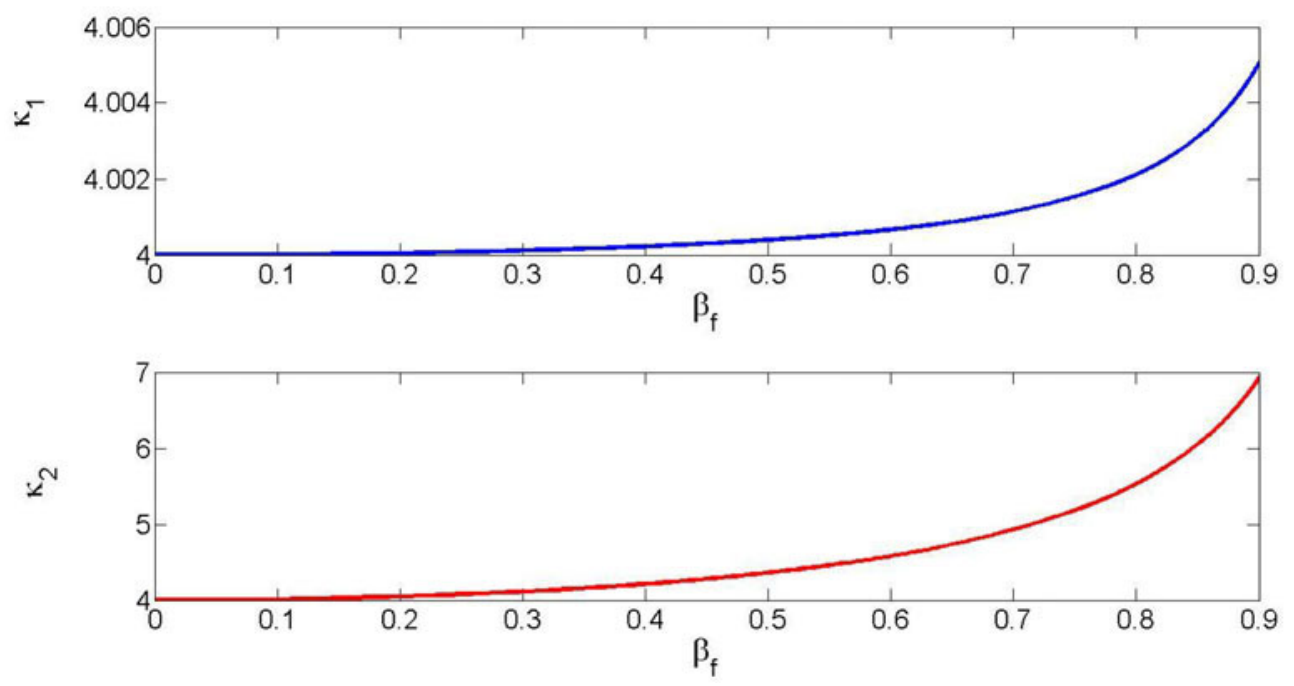

Figure 8. The compressions of the shocked target $\kappa_{1}$ and the shocked flyer $\kappa_{2}$ for $\rho_{0 t} / \rho_{0 f}=K=1000$.
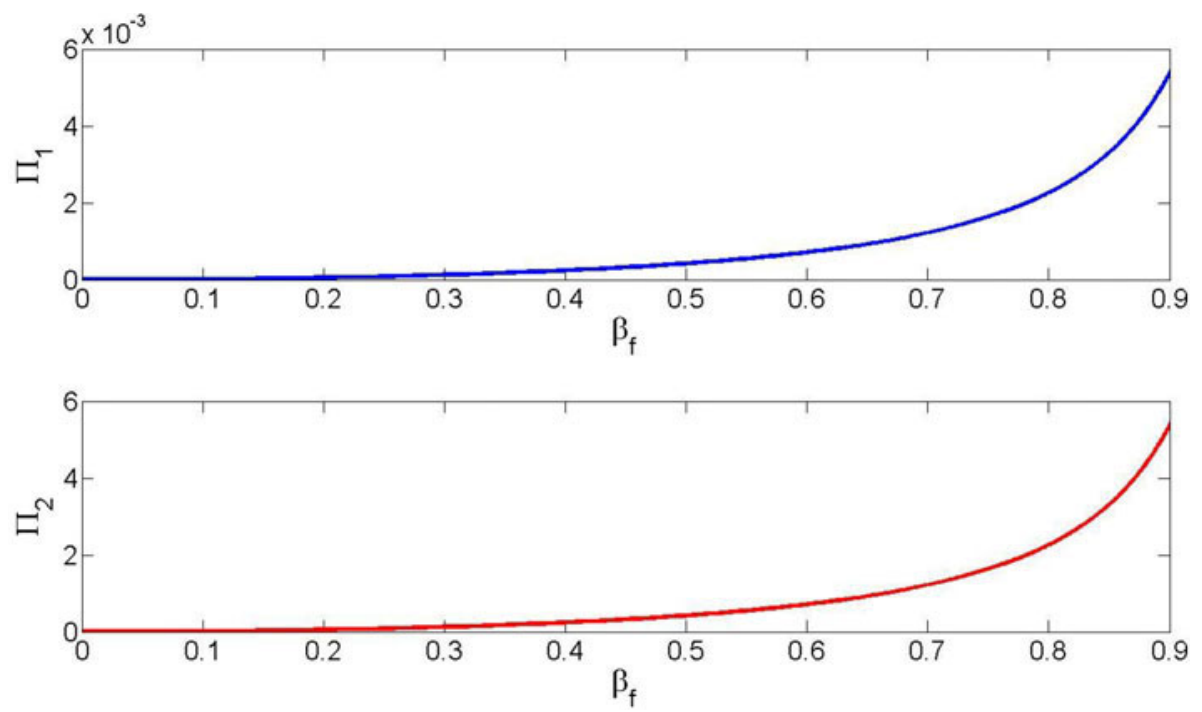

Figure 9. The pressures of the dimensionless shocked target $\Pi_{1}$ and the shocked flyer $\Pi_{2}$ for $\rho_{0 t} / \rho_{0 f}=K=1000$.

$I_{i}(i=1,2)$ are defined in Equations (30). We take $\Gamma_{t}=$ $\Gamma_{f}=5 / 3$ and $\Pi_{i}(i=1,2)$ is given in Equations (29). The solutions of the compressions $\kappa_{1}$ and $\kappa_{2}$ are given in Figure 8, the dimensionless pressures of the shocked target and flyer are given in Figure 9 and the shock velocities $u_{s}$ and particle velocities $u_{p}$ are given in Figure 10, all these figures for $\rho_{0 t} / \rho_{0 f}=K=1000$ (relevant for the fusion ignition case). It is important to note that although the shock wave in the flyer is relativistic, i.e., $u_{s 2} \sim c$, the shock wave in the target is not relativistic, $u_{s 2}<c$, namely $u_{s 2}$ is smaller than $0.05 c$ for a flyer velocity up to $0.9 c$. However, the relativistic formalism is important even for the target since in this case we get a target compression of 4 for finite pressures, of the order of $10^{12}$ bars, while in the non-relativistic formalism when using the ideal gas EOS (like in our case above) one needs an infinite pressure for a compression of 4 .
A crucial question in accelerating a foil to relativistic velocity is its hydrodynamic stability. In particular, the relativistic Rayleigh-Taylor instability was calculated ${ }^{[24,29]}$ as described in the following equations

$$
\begin{gathered}
\xi_{N R}=\frac{\Delta x}{x_{0}}=\exp \left(\frac{t}{\tau_{N R}}\right) \\
\xi_{R}=\frac{\Delta x}{x_{0}}=\exp \left[\left(\frac{t}{\tau_{R}}\right)^{1 / 3}\right] \\
\frac{\tau_{R}}{\tau_{N R}}=\left(\frac{1}{3 \pi}\right)\left(\frac{L}{l_{0}}\right)\left(\frac{I_{L}}{\rho_{0} c^{3}}\right) \\
\frac{1}{\tau_{N R}}=\left[\left(\frac{4 \pi I_{L}}{\rho c l L}\right) \operatorname{tgh}\left(\frac{2 \pi l}{L}\right)\right]^{1 / 2} .
\end{gathered}
$$



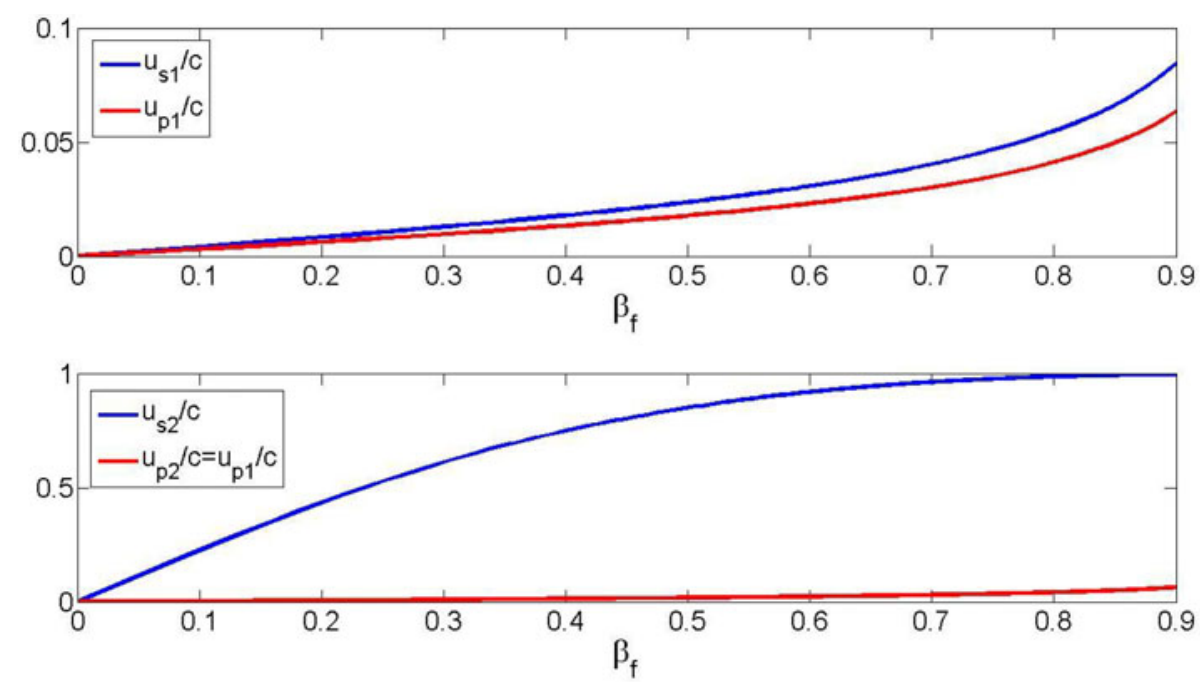

Figure 10. The shock and particle velocities accordingly, $u_{s}$ and $u_{p}$, for $\rho_{0 t} / \rho_{0 f}=K=1000$.

$\xi_{N R}$ and $\xi_{R}$ are accordingly the non-relativistic and relativistic development of the instability for an initial disturbance $x_{0} . L$ is the target dimension orthogonal to the $x$ amplitude and $l$ is the initial foil thickness. We consider the following example: $L=10 \mu \mathrm{m}, x_{0}=10 \mathrm{~nm}, \rho_{0}=1 \mathrm{~g} \mathrm{~cm}^{-3}, l=$ $0.1 \mu \mathrm{m}, I_{L}=10^{24} \mathrm{~W} \mathrm{~cm}^{-2}$. Assuming that the foil breaks for $\xi=10$ (i.e., $\Delta x \sim l$ ) then the foil breaks at $14.2 \mathrm{fs}$ $\left(\tau_{N R}=6.2 \mathrm{fs}\right)$ for the non-relativistic case while in the relativistic regime the foil is stable during $90.5 \mathrm{fs}\left(\tau_{R}=\right.$ $39.3 \mathrm{fs}$ ). This behavior is understood from the different time dependence scaling of the RT instability in relativistic and non-relativistic cases.

\section{The DT fusion ignition}

We analyze the nuclear fusion reactions

$$
\begin{gathered}
A_{1}+A_{2} \rightarrow A_{3}+A_{4}+E_{f}, \\
E_{f}=E_{\alpha}+E_{\text {others }} .
\end{gathered}
$$

$E_{f}$ is the fusion energy in each reaction, $E_{\alpha}$ is $\alpha$ particles energy usually deposited in part into the ignition domain and $E_{\text {others }}$ is the energy contained in the other particles and practically not contained in the ignition volume under consideration. The ignition fusion power $W_{f}\left[\mathrm{erg} /\left(\mathrm{cm}^{3} \mathrm{~s}\right)\right]$ is given by

$$
W_{f}\left[\frac{\mathrm{erg}}{\mathrm{cm}^{3} \mathrm{~s}}\right]=n_{1} n_{2}\langle\sigma v\rangle_{12} E_{\alpha}
$$

where $n_{1}$ and $n_{2}$ are the appropriate densities of particles $A_{1}$ and $A_{2}, \sigma$ is the cross-section of Reaction (33), $\langle\sigma v\rangle_{12}$ is the fusion rate of this reaction and $E_{\alpha}$ is $\alpha$ particles energy.

The equation describing the ignition requirement is given by

$$
W_{f}-\sum W(\text { losses }) \geqslant 0 .
$$

The power density losses, $W$ (losses), include the power densities of the mechanical work $\left(W_{m}\right)$, bremsstrahlung radiation $\left(W_{B}\right)$ and the heat wave transport by electrons $\left(W_{h e}\right)$. Calculating these terms ${ }^{[30]}$ explicitly one gets the ignition criterion for the deuterium (D)-tritium (T) fusion yielding a neutron (n) and a helium nuclei particle $(\alpha)$ : $\mathrm{D}+\mathrm{T} \rightarrow \mathrm{n}+\alpha+17.6 \mathrm{MeV}$

$$
\begin{aligned}
& a\left(T_{e}, T_{i}\right)(\rho R)^{2}+b\left(T_{e}, T_{i}\right)(\rho R)+c\left(T_{e}\right) \geqslant 0, \\
& a\left(T_{e}, T_{i}\right)=8.07 \times 10^{40}\langle\sigma v\rangle_{D T} \\
& -8.63 \times 10^{21} T_{e}(\mathrm{eV})^{1 / 2}\left(1+\frac{2 T_{e}(\mathrm{eV})}{500000}\right), \\
& b\left(T_{e}, T_{i}\right)=-1.02 \times 10^{18}\left[T_{e}(\mathrm{eV})+T_{i}(\mathrm{eV})\right]^{1.5}, \\
& c\left(T_{e}\right)=-\frac{3.11 \times 10^{9} T_{e}(\mathrm{eV})^{7 / 2}}{\ln \Lambda} .
\end{aligned}
$$

The numerical values of Equations (36) are obtained for equal density numbers for deuterium and tritium $n_{D}$ and $n_{T}$, accordingly. $\langle\sigma v\rangle_{D T}$ is the reactivity of the DT reaction fitted in the domain of ion temperatures $1 \mathrm{keV}<T_{i}<$ $100 \mathrm{keV}$ by Ref. [31] given by

$$
\begin{aligned}
&\langle\sigma v\rangle_{D T}\left[\frac{\mathrm{cm}^{3}}{\mathrm{~s}}\right]= 6.4341 \times 10^{-14} \zeta^{-5 / 6}\left(\frac{6.661}{T_{i}^{1 / 3}}\right)^{2} \\
& \times \exp \left[-19.983\left(\frac{\zeta}{T_{i}}\right)^{1 / 3}\right], \\
& \zeta=1-\frac{15.136 T_{i}+4.6064 T_{i}^{2}-0.10675 T_{i}^{3}}{1000+75.189 T_{i}+13.5 T_{i}^{2}+0.01366 T_{i}^{3}} ; \\
& T_{i} \text { in } \mathrm{keV} .
\end{aligned}
$$

The solution of Equations (36) with $\langle\sigma v\rangle_{D T}$ from Equations (37) and $\ln \Lambda=3.5$ is given in Figure 11. Contours of 


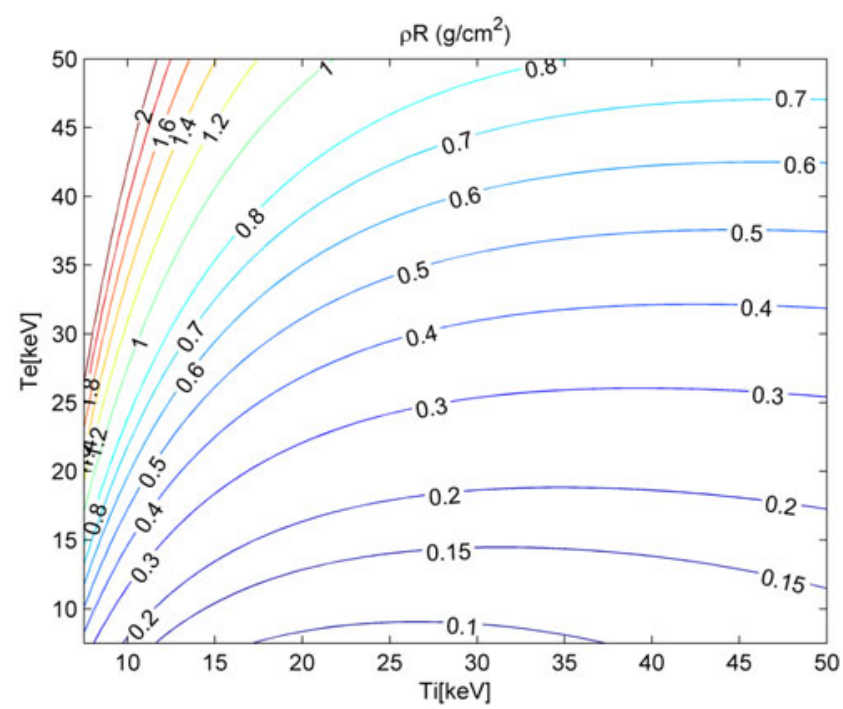

Figure 11. Contours of equal $\rho \cdot R$ as a function of ions and electrons temperatures for DT.

equal $\rho \cdot R$ as a function of ions and electrons temperatures for DT are displayed. It is seen that for temperatures $T_{i}, T_{e}$ in the range $10-50 \mathrm{keV} \rho \cdot R<1$.

In the framework of the piston model, a shock wave is generated in the target, with different ions and electrons temperatures. The time development of the ion and electron temperatures can be derived from the energy conservation Equations (38) coupled to the number density Equation (39),

$$
\begin{gathered}
\left(\frac{3}{2}\right) \frac{d}{d t}\left(n_{e} k_{B} T_{e}\right)=\eta_{d} W_{d}+W_{i e}-W_{B}+f_{\alpha} \eta_{f} W_{f}, \\
\left(\frac{3}{2}\right) \frac{d}{d t}\left(n_{i} k_{B} T_{i}\right)=\left(1-\eta_{d}\right) W_{d}-W_{i e}+f_{\alpha}\left(1-\eta_{f}\right) W_{f}, \\
\frac{d n_{D}}{d t}=\frac{d n_{T}}{d t}=-\frac{d n_{\alpha}}{d t}=-n_{D} n_{T}\langle\sigma v\rangle_{D T},
\end{gathered}
$$

where $W_{d}\left[\mathrm{erg} /\left(\mathrm{cm}^{3} \mathrm{~s}\right)\right]$ is the power density deposited by the laser piston,

$$
\begin{gathered}
\frac{W_{k}}{V}\left[\frac{\mathrm{erg}}{\mathrm{cm}^{3}}\right]=(\gamma-1) \rho c^{2}\left(\frac{t}{\tau_{L}}\right)=\frac{1}{2} \rho u_{p}^{2}\left(\frac{t}{\tau_{L}}\right), \\
W_{d}=\frac{d}{d t}\left(\frac{W_{k}}{V}\right)=\frac{1}{2}\left(\frac{\rho u_{p}^{2}}{\tau_{L}}\right), \\
I_{L}\left[\frac{W}{\mathrm{~cm}^{2}}\right]=4 \times 10^{3} \frac{W_{d}\left[\frac{\mathrm{erg}}{\mathrm{cm}^{3} \mathrm{~s}}\right] \tau_{L}[s]}{\kappa},
\end{gathered}
$$

where $W_{k}$ is the kinetic energy of the flow in the shocked volume and $I_{L}$ is the laser irradiance. In the first equation of Equations (40) $\gamma$ is the relativistic factor defined in Equations (2) and the right hand side of this equation is the non-relativistic limit. $\eta_{d}$ is the fraction of the driver energy deposited in the electrons inside the shocked volume, $\left(1-\eta_{d}\right)$ gives the fraction of the driver energy deposited in the ions inside the shocked volume. $\eta_{d}$ is

$$
\begin{gathered}
\eta_{d}=\frac{\lambda_{i}}{\lambda_{i}+\lambda_{e}} ; \quad E_{i}=\frac{1}{2} m_{i} u_{p}^{2}=1250(\mathrm{MeV})\left(\frac{u_{p}}{c}\right)^{2}, \\
\lambda_{i}[\mathrm{~cm}]=\left(\frac{3 \times 10^{23}}{n_{i}}\right)\left(\frac{m_{p}}{m_{i}}\right) E_{i}[\mathrm{MeV}], \\
\lambda_{e}[\mathrm{~cm}]=\left(\frac{5 \times 10^{22}}{n_{e} \ln \Lambda}\right) T_{e}[\mathrm{keV}]^{3 / 2} E_{i}[\mathrm{MeV}],
\end{gathered}
$$

where $\lambda_{i}$ and $\lambda_{e}$ are the appropriate mean free paths of the ions and electrons in plasma.

$W_{i e}\left[\mathrm{erg} /\left(\mathrm{cm}^{3} \mathrm{~s}\right)\right]$ is the ion-electron exchange power density,

$$
\begin{gathered}
W_{i e}\left[\frac{\mathrm{erg}}{\mathrm{cm}^{3} \mathrm{~s}}\right]=\left(\frac{3}{2}\right) \frac{k_{B}\left(T_{i}-T_{e}\right)}{\tau_{e q}}, \\
\tau_{e q}=\frac{3 m_{e} m_{i}}{8 \sqrt{2 \pi} n_{i} e^{4} \ln \Lambda}\left(\frac{k_{B} T_{e}}{m_{e}}+\frac{k_{B} T_{i}}{m_{i}}\right)^{3 / 2},
\end{gathered}
$$

$W_{B}\left[\mathrm{erg} /\left(\mathrm{cm}^{3} \mathrm{~s}\right)\right]$, the electron bremsstrahlung power density losses

$W_{B}\left[\frac{\mathrm{erg}}{\mathrm{cm}^{3} \mathrm{~s}}\right]=8.58 \times 10^{21} \rho^{2} T_{e}(e V)^{0.5}\left(1+\frac{2 T_{e}(\mathrm{eV})}{0.511 \times 10^{6}}\right)$,

and $W_{f}\left[\mathrm{erg} /\left(\mathrm{cm}^{3} \mathrm{~s}\right)\right]$, the fusion power density created in the shocked volume. $\eta_{f}$ is the energy fraction that is deposited in the electrons by the $\alpha$-particles created in the fusion under consideration and $\left(1-\eta_{f}\right)$ describes the energy fraction that is deposited in the ions by these $\alpha$-particles. $\eta_{f}$ is $^{[32]}$

$$
\eta_{f}=\frac{32}{32+T_{e}(\mathrm{keV})} .
$$

$f_{\alpha}$ is the fraction of the $\alpha$-particles created and deposited into the ignitor domain, while $\left(1-f_{\alpha}\right)$ is the escape fraction to the surrounding cold fuel. $f_{\alpha}$ is given by

$$
\begin{gathered}
f_{\alpha}=\left\{\begin{array}{lc}
\frac{3}{2} x_{\alpha}-\frac{4}{5} x_{\alpha}^{2} & x_{\alpha}<\frac{1}{2} \\
1-\frac{1}{4 x_{\alpha}}+\frac{1}{160 x_{\alpha}^{3}} & x_{\alpha} \geqslant \frac{1}{2},
\end{array}\right. \\
x_{\alpha}(\tau)=\frac{R}{R_{\alpha}} ; \quad R=\left(u_{s}-u_{p}\right) \tau_{L}, \\
R_{\alpha}[\mathrm{cm}]=\frac{1}{\kappa \rho_{0}}\left[\frac{1.5 \times 10^{-2} T_{e}(\mathrm{keV})^{5 / 4}}{1+8.2 \times 10^{-3} T_{e}(\mathrm{keV})^{5 / 4}}\right] .
\end{gathered}
$$

Figure 12 display the results of the two temperatures model for DT pre-compressed to density $\rho_{0}=600 \mathrm{~g} \mathrm{~cm}^{-3}$. The fast ignition shock generated by irradiation with laser intensity of $7.5 \times 10^{22} \mathrm{~W} \mathrm{~cm}^{-2}, 1 \mathrm{ps}$ pulse duration and energy $3.67 \mathrm{~kJ}$ (see Equation (16)) induces a compression of $\kappa=4$. 


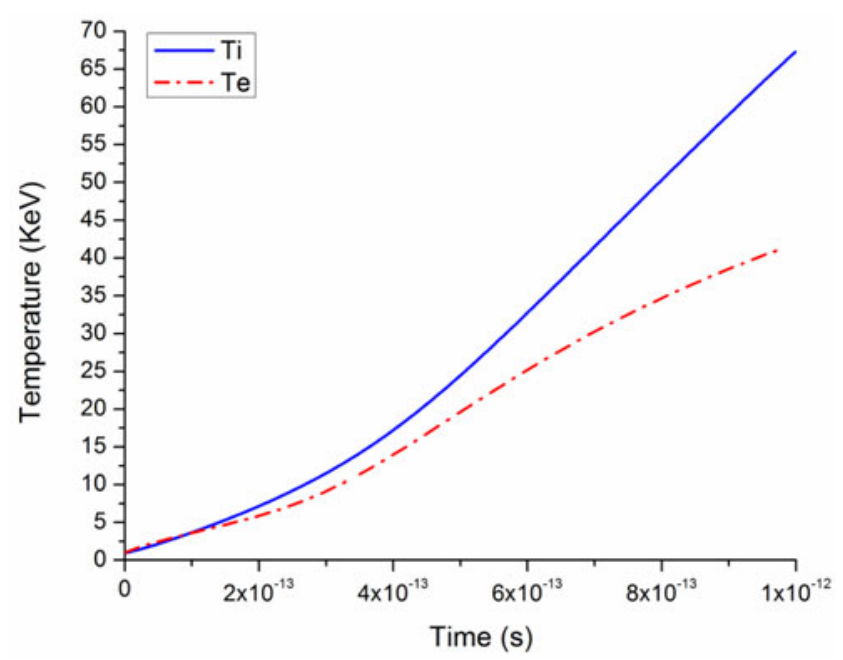

Figure 12. Electrons $T_{e}$ and protons $T_{i}$ temperatures as a function of time for a DT case satisfying the ignition criterion.

\section{Laser induced fusion detonation wave}

Recently $^{[33]}$ a self-sustained 1D detonation wave was suggested to be possible due to the heating by the alpha particles generated in the laser-induced ignitor. This detonation wave should sustain ignition in the remaining part of the target. This fast ignition scheme is schematically described in Figure 14. The ignitor operation was calculated in Section 4. For the detonation we chose a laser yielding a particle velocity behind the shock front of $1.0 \%$ the speed of light. The theoretical treatment that we consider is based on 1D plane detonation wave under Chapman-Jouguet (CJ) condition.

In the case of chemical-based detonation, the energetic material entering the shock front is compressed and thus its temperature rises. Under sufficient temperature the material transforms exothermally into gaseous products releasing energy per unit mass $(Q)$ that supports the shock. The governing parameter effecting reaction rate in the reaction zone are the local density and temperature. Our detonation is analogous to this description where the chemical energy has been changed to nuclear fusion energy. In the DT fusion one gets $17.6 \mathrm{MeV}$ fusion energy per reaction but only the $3.52 \mathrm{Mev}$ of the $\alpha$ particle is relevant to support the desired steady state shock condition.

By using CJ formalism for the ideal gas case one can obtain from the conservation equations the following useful relations

$$
\begin{gathered}
\left(\frac{\rho}{\rho_{0}}\right)_{C J}=\frac{\Gamma+1}{\Gamma} ; \quad P_{C J}=\frac{\rho_{0} D^{2}}{\Gamma+1}, \\
\frac{u}{D}=\frac{1}{\Gamma+1} ; \quad \frac{c_{s}}{D}=\frac{\Gamma}{\Gamma+1}, \\
\frac{Q}{D^{2}}=\frac{1}{2\left(\Gamma^{2}-1\right)},
\end{gathered}
$$

where $P\left[\mathrm{erg} \mathrm{cm}^{-3}\right]$ is the pressure, $\rho\left[\mathrm{g} \mathrm{cm}^{-3}\right]$ is the density, $E_{T}$ [ $\mathrm{erg} \mathrm{g}^{-1}$ ] is the thermal energy, $Q$ [ $\mathrm{erg} \mathrm{g}^{-1}$ ] is the nuclear fusion energy deposited on the wave front, $u$ is the velocity of motion of the fluid [ $\left.\mathrm{cm} \mathrm{s}^{-1}\right]$ and $D\left[\mathrm{~cm} \mathrm{~s}^{-1}\right]$ is the detonation wave velocity. The detonation wave is steadily propagating with velocity $D$, namely all magnitudes $P, \rho, u$ and $E_{T}$ are functions of time $t$ and space $x$ only in the form $x-D t$. For the detonation wave we take ${ }^{[34]} \Gamma=3$ implying

$$
\frac{u}{D}=\frac{1}{4} ; \quad \frac{c_{s}}{D}=\frac{3}{4} ; \quad \frac{Q}{D^{2}}=\frac{1}{16} .
$$

We can see that by determining the particle velocity $u=u_{p}$ to equal $1.0 \%$ the speed of light we have determined the nuclear energy needed to support a steady state $\mathrm{CJ}$ condition, $Q=9 \times 10^{12} \mathrm{~J} \mathrm{~kg}^{-1}$. As one can see from Figure 15 this value of $Q$ is achieved by our laser-induced detonator, where $Q$ in our model was calculated from

$$
Q\left[\frac{\mathrm{J}}{\mathrm{kg}}\right]=\left(\frac{E_{\alpha}}{\rho}\right) \int_{0}^{t=\tau_{L}} d t\left(\frac{d n_{\alpha}}{d t}\right) \frac{1}{2}\left(1+f_{\alpha}\right),
$$

where $f_{\alpha}$ is defined in Equations (45). In this case the nuclear fusion ignition conditions for the pre-compressed DT plasma are achieved along the detonation wave orbit.

\section{Summary and perspective}

In the physical domain where relativistic shock waves are generated, mechanical interactions fully dominate over thermal phenomena, because there is no time for thermal relaxation and expansion. As the laser photons momentum is fully collimated, mechanical interactions are very much obliged to follow that direction. On the contrary, fusion ignition onset requires the thermal interactions to dominate, although supra-thermal reactions can have a complementary role. Such a conversion from a regime to another is reached by a head-on crash, for instance, between two opposite shock waves in the center of a pre-compressed micro-target.

The physics of inertial confinement fusion (ICF) is based on compressing and igniting the plasma fuel ${ }^{[32,35,36]}$. In order to ignite the fuel with less energy it was suggested to separate the drivers that compress and ignite the target ${ }^{[37,38]}$. This idea is called fast ignition. Many schemes have been suggested to solve this issue ${ }^{[39]}$.

In the previous section we described the condition of a shock wave ignition scheme, where the ignition shock wave is generated directly by the high irradiance laser or indirectly by the impact of a laser accelerated micro-foil. This shock wave is created in a pre-compressed target that was irradiated by many laser beams. This fast ignition scheme induced by the impact of a micro-foil is schematically described in Figure 13. 
(a)

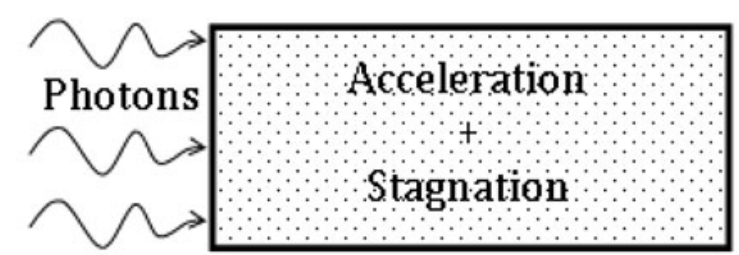

(b)

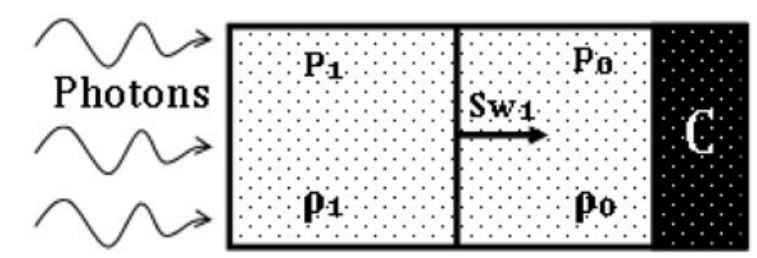

(c)

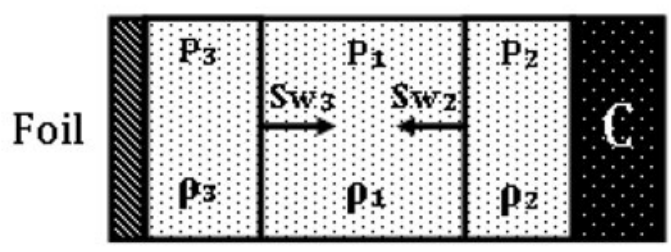

(d)

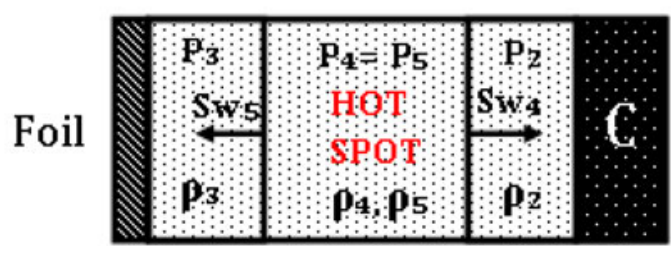

Figure 13. The fast ignition scheme by the impact of a high irradiance laser accelerated foil. (a) The pre-compression by the nanosecond laser beams. (b)-(d) The sequence of shock waves leading to the ignition hot spot.

The directly laser-induced shock wave velocity is in the intermediate domain between the relativistic and nonrelativistic hydrodynamics as termed in Section 2 of this paper. The laser intensities apply a ponderomotive force that forms a $\mathrm{DL}^{[40]}$ which acts as a piston driving this shock wave moving in the pre-compressed target. This laser is described in the literature as a 'piston model' $[21,24,25]$.

In this paper we calculate the high velocities achieved by the laser acceleration of a micro-foil. This high velocity foil collides with a second foil resulting in the creation of the relativistic shock waves. The analytically derived thermodynamic parameters in these collisions are enormous and they might exist only in astrophysical phenomena or nuclear collisions.

Due to the recent developments in high power lasers in the multi Petawatt domain it is also suggested in this paper to accelerate micro-foils to relativistic velocities. From Figure 6 we learn that one can get a micro-foil with half the speed of light for a laser pulse duration $\tau$ if $I \tau=$ $4.5 \times 10^{8} \mathrm{~J} \mathrm{~cm}^{-2}$ (e.g., 0.5 fs laser with $I=10^{24} \mathrm{~W} \mathrm{~cm}^{-2}$ ) for a flyer with initial density $1 \mathrm{~g} \mathrm{~cm}^{-3}$ and a foil thickness of $0.1 \mu \mathrm{m}$. A cross-section area of $10 \mu \mathrm{m}^{2}$ will require laser energy of $45 \mathrm{~J}$.

Furthermore, we suggest measuring experimentally the flyer velocity using the relativistic DE, while the initial density could be estimated experimentally using an x-ray pulse created by a secondary laser beam.

Taking into account that during few femtoseconds one can accelerate a micro-foil to relativistic velocities one can achieve stable relativistic acceleration for laser irradiances of the order of $10^{24} \mathrm{~W} \mathrm{~cm}^{-2}$.

The laser energy can be reduced significantly if we use two lasers in order to accelerate two micro-foils in opposite directions, thus causing them to collide. According to the law of the addition of relativistic velocities, the relative impact velocity between the foils is given by $\left(\beta_{1}+\beta_{2}\right) /\left(1+\beta_{1} \beta_{2}\right)$. For example, if $\beta_{1}=\beta_{2}=0.8$ then the impact relative velocity is $0.9756 c$; implying a very large reduction of energy.

Today particles must be accelerated along many kilometers in order to achieve high energies. In our scheme the foils

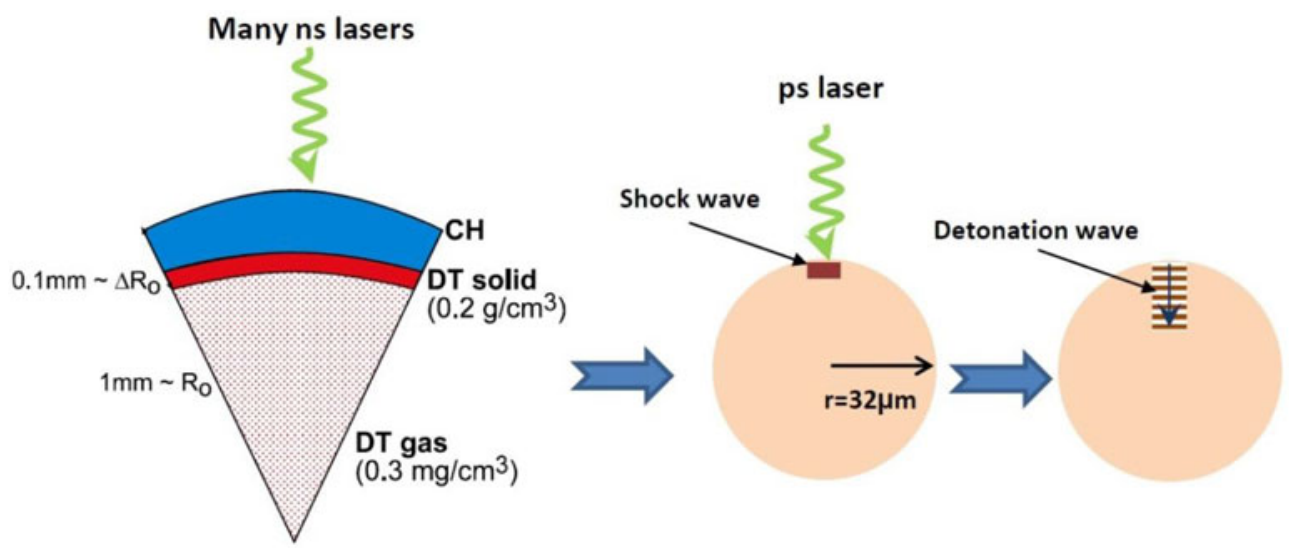

Figure 14. The fast ignition scheme of a detonation wave. 

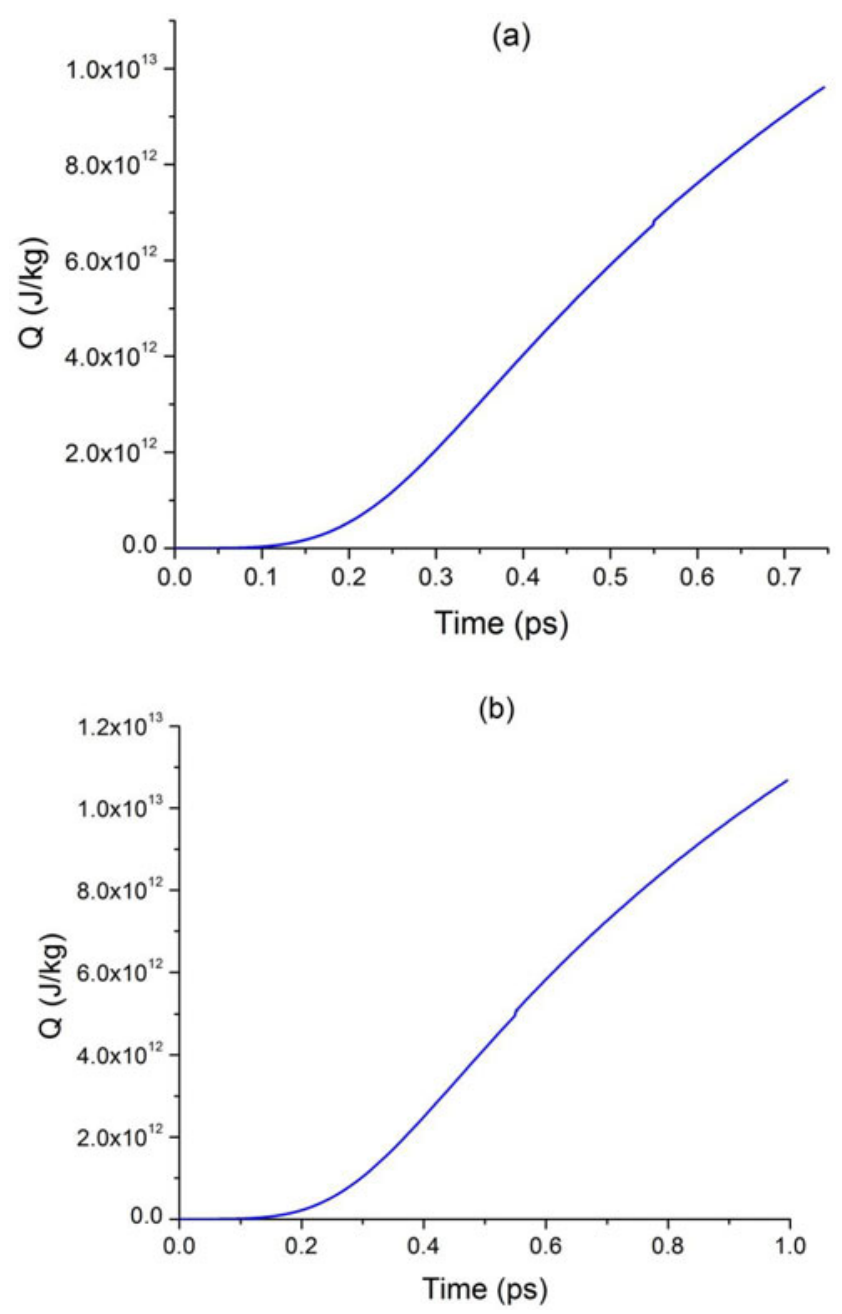

Figure 15. The fusion energy $Q$ per unit mass released in the shock wave forward direction as a function of time in the shocked volume for (a) $\Gamma=3$ and (b) $\Gamma=5 / 3$.

are accelerated to relativistic velocities through a distance of the order of $1 \mathrm{~mm}$ or less. We propose that these relativistic collisions of two micro-foils accelerated by future lasers may offer a new way of accelerating particles or nuclei in the laboratory.

\section{References}

1. P. H. Hugoniot, $1^{e}$ Partie. J. Ecole Polytech. (Paris) 58, 1 (1887) (Translated in reference 2).

2. J. N. Johnson and R. Cheret, Classic Papers in Shock Compression Science (Springer, 1998).

3. Y. B. Zeldovich and Y. P. Raizer, Physics of Shock Waves and High Temperature Hydrodynamic Phenomena (Academic Press Publications, 1966).

4. S. Eliezer and R. A. Ricci, (Eds.) High Pressure Equation of State: Theory and Application, Enrico Fermi School CXIII 1989 (North-Holland, 1991).
5. S. Eliezer, A. K. Ghatak, H. Hora, and E. Teller, Fundamental of Equation of State (World Scientific, 2002), Previously published as S. Eliezer, A. K. Ghatak, H. Hora and E. Teller, Introduction to Equations of State: Theory and Applications (Cambridge University Press, 1986).

6. S. Eliezer, The Interaction of High-Power Lasers with Plasmas (CRC press, 2002).

7. S. Eliezer, in Laser-Plasma Interactions and Applications, P. McKenna, D. Neely, R. Bingham and D. A. Jaroszynski (eds) 68th Scottish Universities Summer School in Physics, (Springer Publication, 2013), p. 49.

8. C. Danson, D. Hillier, N. Hopps, and D. Neely, High Power Laser Sci. Eng. 3, e3 (2015).

9. The ELI project homepage is http://wwwextreme-light-infrast ructure.eu/.

10. L. D. Landau and E. M. Lifshitz, Fluid Mechanics, 2nd edn (Pergamon Press, 1987).

11. A. H. Taub, Phys. Rev. 74, 328 (1948).

12. V. E. Fortov and I. V. Lomonosov, Shock Waves 20, 53 (2010).

13. R. Cauble, D. W. Phillion, T. J. Hoover, N. C. Holmes, J. D. Kilkenny, and R. W. Lee, Phys. Rev. Lett. 70, 2102 (1993).

14. H. Azechi, T. Sakaiya, T. Watari, M. Karasik, H. Saito, K. Ohtani, K. Takeda, H. Hosoda, H. Shiraga, M. Nakai, K. Shigemori, S. Fukoda, M. Murakami, H. Nagamoto, T. Johzaki, J. Gardner, D. G. Colombant, J. W. Bates, A. L. Velikovich, Y. Aglitsky, J. Weaver, S. Obenschain, S. Eliezer, R. Kodama, T. Norimatsu, H. Fujita, K. Mima, and H. Kan, Phys. Rev. Lett. 102, 235002 (2009).

15. H. Hora, Physics of Laser Driven Plasmas (John Wiley, 1981).

16. R. Sauerbrey, Phys. Plasmas 3, 4712 (1996).

17. H. Hora, J. Badziak, M. N. Read, Y. T. Li, T. J. Liang, H. Liu, Z. M. Shang, J. Zhang, F. Osman, G. H. Miley, W. Y. Zhang, X. T. He, H. S. Peng, S. Glowacz, S. Jablonski, J. Wolowski, Z. Skladanowski, K. Jungwirth, K. Rohlena, and J. Ullschmied, Phys. Plasmas 14, 072701 (2007).

18. P. Lalousis, H. Hora, S. Eliezer, J. M. Martinez-Val, S. Moustaizis, G. H. Miley, and G. Mourou, Phys. Lett. A 377, 885 (2013).

19. H. Hora, Laser Particle Beams 30, 325 (2012).

20. P. Lalousis, I. Földes, and H. Hora, Laser Particle Beams 30, 233 (2012).

21. T. Esirkepov, M. Borghesi, S. V. Bulanov, G. Mourou, and T. Tajima, Phys Rev. Lett. 92, 175003 (2004).

22. P. L. Robinson, M. Zepf, S. Kar, R. G. Evans, and C. Bellei, New J. Phys. 10, 013021 (2008).

23. G. Marx, Nature 211, 22 (1966).

24. S. Eliezer, N. Nissim, J. M. Martinez Val, K. Mima, and H. Hora, Laser Particle Beams 32, 211 (2014).

25. N. Naumova, T. Schlegel, V. T. Tikhonchuk, C. Labaune, I. V. Sokolov, and G. Mourou, Phys. Rev. Lett 102, 025002 (2009).

26. S. Eliezer, N. Nissim, E. Raicher, and J. M. Martinez Val, Laser Particle Beams 32, 243 (2014).

27. S. Eliezer, N. Nissim, V. S. Pinhasi, E. Raicher, and J. M. Martinez Val, High Power Laser Sci. Eng. 2, e22 (2014) .

28. S. Eliezer, J. M. Martinez Val, and S. V. Pinhasi, Laser Particle Beams 31, 113 (2013).

29. F. Pegoraro and S. V. Bulanov, Phys. Rev. Lett 99, 065002 (2007). 
30. S. Eliezer, Z. Henis, N. Nissim, S. V. Pinhasi, and J. M. Martinez Val, Laser Particle Beams 33, 577 (2015).

31. H. S. Bosch and G. M. Hale, Nuclear Fusion 32, 611 (1992).

32. S. Atzeni and J. Meyer-Ter-Vehn, The Physics of Inertial Fusion (Claredon Press, 2004).

33. S. Eliezer, A. Ravid, Z. Henis, N. Nissim, and J. M. Martinez Val, Laser Particle Beams 34, 343 (2016).

34. L. D. Landau and K. P. Stanyukovich, Dokl. 46, 339 (1945).

35. J. H. Nuckolls, L. Wood, A. Thiessen, and G. B. Zimmermann, Nature 239, 139 (1972).
36. G. Velarde and N. Carpintero-Santamaria, (Eds.) Inertial Confinement Nuclear Fusion: A Historical Approach by its Pioneers (Foxwell and Davies Pub., 2007).

37. N. G. Basov, S. Y. Guskov, and L. P. Feoktistov, J. Soviet Laser Res. 13, 396 (1992).

38. M. E. Tabak, J. Hammer, M. E. Glinsky, W. L. Kruer, S. C. Wilks, J. Woodworth, E. M. Campbell, M. D. Perry, and R. J. Mason, Phys. Plasmas 1, 1626 (1994).

39. S. Y. Guskov, Plasma Phys. Rep. 39, 1 (2013).

40. H. Hora, P. Lalousis, and S. Eliezer, Phys. Rev. Lett. 53, 1650 (1984). 